\title{
Stability in coalition formation games
}

\author{
Katarína Cechlárová ${ }^{\text {, Antonio Romero-Medina }}{ }^{2}$ \\ 'Department of Geometry and Algebra, P. J. Šafárik University, Jesenná 5, 04154 Košice, \\ Slovakia (e-mail: cechlarova@duro.upjs.sk) \\ ${ }_{2}^{2}$ Departamento de Economia, Universidad Carlos III, 28903 Getafe, Spain \\ (e-mail: aromero@eco.uc 3 m.es)
}

\begin{abstract}
In the context of coalition formation games a player evaluates a partition on the basis of the set she belongs to. For this evaluation to be possible, players are supposed to have preferences over sets to which they could belong. In this paper, we suggest two extensions of preferences over individuals to preferences over sets. For the first one, derived from the most preferred member of a set, it is shown that a strict core partition always exists if the original preferences are strict and a simple algorithm for the computation of one strict core partition is derived. This algorithm turns out to be strategy proof. The second extension, based on the least preferred member of a set, produces solutions very similar to those for the stable roommates problem.
\end{abstract}

Key words: Coalition formation games, core, stable roommates problem, digraphs, algorithms.

\section{JEL classification: C78, D71, D78}

\section{Introduction}

For the classical stable roommates problem not every instance has a stable solution. One possibility for avoiding this difficulty is to restrict the set of

1991 Mathematics Subject Classification. 90D06, 90D12

Acknowledgement. The authors would like to express their gratitude to J. E. Martinez Legaz, David Perez Castrillo and Jose Alcalde for valuable and stimulating discussions. We also thank one of the anonymous referees for drawing our attention to the existing literature in this field.

1 The work of this author was supported by the Slovak Grant Agency for Science under contract "Combinatorial Structures and Complexity of Algorithms" 
considered preferences, as was studied by Alcalde [1]. Another approach was suggested by Romero-Medina [9] in the sense that not only pairs, but also groups of different cardinality are allowed. The outcome of such a game is now a partition of the set of players.

A coalition formation game is an $n$-player game with outcomes being partitions of the player set $N=\{1,2 \ldots n\}$. A player $i$ evaluates the possible outcome $\mathscr{H}$ according to his valuation of the set $M(i)$ she belongs to. A coalition $Z$ of players could profitably deviate from the existing partition $\mathscr{H}$ if each member $i$ of $Z$ prefers $Z$ strictly to $M(i)$; or if each player $i$ in $Z$ either prefers $Z$ before $M(i)$ strictly or is indifferent between the two sets and at least one player strictly prefers $Z$ before her set in $\mathscr{M}$. If there is no coalition with a tendency to deviate, the existing partition is in a sense stable; in the game theoretic literature such outcomes are said to be in the core or in the strict core of the game respectively (compare the results of Roth and Postlewaite [11]).

Usually it is supposed that each player ranks the sets to which she could belong. In [2] and [4], the authors impose various conditions on the possible rankings (anonymity, single peakedness, top coalition property etc) and study which of them ensure the existence of a core partition. Our approach is different. We start with players having preferences over other (individual) players and then consider two possibilities of extending the preferences over individuals to preferences over groups of players. In the first case (מB-preferences, Section 3), a player ranks the sets according to her most preferred member of the set and to the size of the set. For such preferences a strict core partition exists for any instance of the problem, if all the preferences are strict. One such partition can be found by an algorithm, which is almost identical with the top-trading cycles algorithm of David Gale (see Shapley and Scarf [13]). This algorithm is moreover strategy-proof in the sense that no player and even no coalition of players can profitably misrepresent their preferences to obtain a better outcome (see Roth [10], Bird [3]).

In the second case ( $\mathscr{H}$-preferences, Section 4 ), the preferences over sets are derived from preferences over the least preferred member of the set. We show that if all the preferences are strict, then this model is very similar to the stable roommates problem.

\section{Definitions}

Let $N=\{1,2, \ldots, n\}$ be the set of players. We suppose that each player $i$ has preferences over individual players, represented by a complete, reflexive and transitive binary relation $\leqslant_{i}$ on $N$. Strict preference relation and indifference relation of player $i$ are denoted by $\prec_{i}$ and $\sim_{i}$ respectively. A player $j$ 'such that $j \prec_{i} i$ is for $i$ unacceptable, the other players are acceptable.

A preference profile $\left(\leqslant_{1}, \leqslant_{2}, \ldots, \leqslant_{n}\right)$ will be denoted by $\mathscr{P}$. If $S$ is any subset of $N$, then by $\mathscr{P}_{S}$ we denote the restriction of the preference profile $\mathscr{P}$ to the set $S$.

We shall consider partitions of the set $N$ of the form $\mathscr{M}=\left(M_{1}, M_{2}, \ldots\right.$ $M_{r}$ ), where $M_{p}$ and $M_{q}$ are disjoint if $p \neq q$ and the union of $M_{1}, \ldots, M_{r}$ is equal to $N$. The set in this partition, containing $i$, will be denoted by $M(i)$.

Definition 1. A coalition formation game is a pair $(N, \mathscr{P})$. 
In a coalition formation game the players evaluate the outcomes, i.e. partitions, according to their preferences over sets, to which they belong. We start with players having preferences over other individual players and suppose that their preferences over sets are derived from those individual preferences. Before we introduce two such set-preferences, we define the solution concept used. So far, the same symbols are used to denote the preferences of players over individuals as well as over sets.

Definition 2. A coalition $Z \subseteq N$ weakly blocks a partition $\mathscr{M}$ if for each $i \in Z$ it holds $Z \succcurlyeq_{i} M(i)$ and for at least one $j \in Z$ we have $Z \succ_{j} M(j)$. A partition which admits no weakly blocking coalition is called a strict core partition.

Let us remark here that a blocking coalition $Z$ is such that each $i \in Z$ strictly prefers $Z$ over $M(i)$ and core partitions admit no blocking coalition (compare Roth and Postlewaite [11]). Notice that our results ensuring the existence of strict core partitions naturally imply also the existence of core partitions, since when no weakly blocking coalition exists, there is also no blocking coalition.

Definition 3. Let $i \in S . S \subseteq N$, denote by $S^{\prime}=S-\{i\} . \mathscr{B}_{i}(S)$ is any player $j \in S$, such that $j \geqslant_{i} k$ for all $k \in S . \mathscr{W}(S)$ is any player $j \in S^{\prime}$, such that $j \leqslant i k$ for all $k \in S^{\prime}$; if $S=\{i\}$ then we set $\mathscr{W}_{i}(S)=i$. (M standing for 'best' and $\mathscr{W}$ for 'worst', respectively.)

Definition 4. A set $S$ is $\mathscr{B}$-preferred by $i$ over a set $T$ (written $S \succ \mathscr{B , i} T$ ) if

(1) $\mathscr{B}_{i}(S) \succ_{i} \mathscr{B}_{i}(T)$ or

(2) $\mathscr{B}_{i}(S) \sim_{i} \mathscr{B}_{i}(T)$ and $|S|<|T|$.

Definition 5. A set $S$ is $\mathscr{W}$-preferred by $i$ over a set $T$ (written $S \succ \mathscr{W}, T$ ) if $\mathscr{W}_{i}(S) \succ_{i} \mathscr{W}_{i}(T)$

Clearly, $\{i\}$ is $\mathscr{B}$-preferred by $i$ over sets containing only unacceptable players and is $\mathscr{B}$-preferred less than sets containing at least one player $j$ such that $j \succ_{i} i$. On the other hand, player $i \mathscr{W}$-prefers $\{i\}$ over sets containing at least one unacceptable player and $\mathscr{W}$-prefers $\{i\}$ less than sets which contain only such players $j$, that $j \succ_{i} i$.

According to the set preferences used, we shall now speak about the $\mathscr{B}$ strict core partitions and the $\mathscr{W}$-strict core partitions. To denote the setpreferences of a player $i$, the symbols $\succ$ and $\geqslant$ will be accompanied by index $i$ as well as by symbols $\mathscr{B}$ or $\mathscr{W}$ respectively, according to the model used.

Definition 4 represents in some sense an 'optimistic' approach, when a player looks only at her most preferred member of a set and does not care about the rest explicitly, only through the size of the set. Notice, that if condition (2) were not present, the grand coalition would be a strict core partition for any preference profile. Definition 5 is on the other hand a 'pesimistic' approach, representing players who are trying to avoid people whom they do not like.

In Section 3 we show that there exists a $\mathscr{B}$-strict core partition for every coalition formation game in which all the preferences are strict and propose a simple mechanism that finds such a partition. In Section 4 it is shown that in 
the case with no indifferences the $\mathscr{W}$-strict core partition problem is closely related to the stable roommates problem.

\section{Properties of $\mathscr{B}$-strict core partitions}

In our study of $\not{B}$-strict core partitions we shall use some notions and facts from graph theory.

A digraph is a pair $G=(V, E)$, where $V$ is the set of vertices and $E$ is the set of arcs, i.e. ordered pairs of vertices. If $e=(i, j) \in E$, we say that $j$ is the head of $e$ and $i$ its tail. Cardinality of the set $\{j ;(i, j) \in E\}$ is called the outdegree of vertex $i$. A sequence $\left(x_{0}, x_{1}, \ldots, x_{r}\right)$ is a path, if $\left(x_{0}, x_{1}\right),\left(x_{1}, x_{2}\right), \ldots$, $\left(x_{r-1}, x_{r}\right)$ are arcs in $G$; a path with $x_{0}=x_{k}$ is a cycle. A path leading to a cycle $\mathscr{b}$ is called a tail of this cycle, the vertices on the cycle $\mathscr{C}$ and on all its tails are said to form the attraction set of $\mathscr{C}$

Let us define for any restriction $p_{S}$ of the preference profile $\not p$ the first preferences digraph $G\left(\mathscr{P}_{S}\right)=(V, E)$ by $V=S$ and $(i, j) \in E$ if and only if $j=\mathscr{B}_{i}(S)$. If all the preferences are strict in the preference profile $\mathscr{P}$, then in the first preferences digraphs $G\left(\mathscr{P}_{S}\right)$ for all the profile restrictions $\mathscr{P}_{S}$ all the vertices have outdegree 1. Such digraphs are simply collections of disjoint attraction sets of several cycles. Moreover, if we start in any vertex of the attraction set of a cycle $\mathscr{C}$ and follow a (unique) path starting in this vertex, we eventually end up in $\mathscr{C}$. This observation leads to the following algorithm. In this algorithm, each repetition of Steps 2 to 4 is callęd a round, the variable $r$ counts the number of rounds. The partition that is obtained is denoted by $\mathscr{M}=\left(M_{1}, M_{2}, \ldots, M_{r}\right)$

Algorithm BSTABLE.

Input: A preference profile ip

Output: A 3 -strict core partition $\mathscr{H}$.

Step I. Set $r:=0, V:=N$

Step 2. If $V=\varnothing$, end. Else choose a player $i_{0}$ randomly from $V$.

Step 3. $i_{0}$ proposes to the first player in her preference list, $i_{1}, i_{1}$ proposes to the first player in her preference list, $i_{2}$ etc, until a player in this chain proposes to somebody, who has already made a proposal in this round. That means, that among proposing players a cycle $/ 6$ has emerged.

Step 4: Set $r:=r+1, M_{r}:=\mathscr{r}, V:=V-\mathscr{r}$ and construct the restricted preference profile $: p_{1}$ Set $\mathscr{P}=\mathscr{P}_{V-\mathscr{r}}$. Go to Step 2

Let us remark here that algorithm BSTABLE is almost identical with the Top-trading-cycles algorithm of David Gale (see [13] or $[10]$ ). This algorithm has been used to find a solution in the houseswapping game in the sense that the traders exchange their houses according to the cycles obtained. Our model is however different, so we must prove the correctness of this algorithm for its use in the $\mathscr{B}$-strict core partitions context separately.

Theorem 1. Let $\mathscr{P}$ be any preference profile with all preferences strict. Then algorithm BSTABLE generates a $\mathscr{B}$-strict core partition. Moreover, the obtained partition is unique, independently from the random choices in Step 2. Hence, a $\mathscr{B}$-strict core partition exists for every preference profile $\mathscr{P}$, if all the preferences are strict. 
Proof: The uniqueness of the outcome results from the structure of the first preferences digraph for strict preferences.

We still have to show that the obtained partition is in $\not B$-strict core. We shall proceed by induction on the number of players. Let $\mathscr{C}=\left(i_{0}, i_{1}, \ldots, i_{p-1}\right)$ be the first cycle obtained in the course of the algorithm. Suppose that $Z$ is a blocking set such that $\mathscr{C} \cap Z \neq \varnothing$. Take an arbitrary $i_{k} \in Z \cap \mathscr{C}$. Then $Z \succeq i_{i_{k}} \mathscr{C}$, which is only possible when $i_{k+1} \in Z$ (indices are understood modulo $p$, if necessary). This implies that then $\mathscr{C} \subseteq Z$. However, should $\mathscr{C}$ be a proper subset of $Z$, then members of $\mathscr{C}$ would strictly prefer $\mathscr{E}$ to $Z$. Hence we obtained that $\mathscr{G} \cap Z \neq \varnothing$ implies $\mathscr{G}=Z$, which is a contradiction. Therefore the members of $\mathscr{C} \in \mathscr{M}$ cannot belong to any coalition that weakly $\mathscr{B}$ blocks $\mathscr{M}$.

Now it is possible to reduce the original preference profile $\mathscr{P}$ to its restriction $\mathscr{P}_{N-\mathscr{H}}$. Since the number of players in $N-\mathscr{C}$ is smaller, the induction hypothesis applies and there cannot be any weakly blocking coalition for $\not 1$

Let us stress here that if the preferences are not strict, it may happen that the problem has no $B$-strict core. Also, the uniqueness of the outcome of the algorithm is in the case with indifferences not ensured. This is illustrated by the following example.

Example 1. Suppose we are given the following preférence profile.

$$
\begin{aligned}
& 5 \succ_{1} 3 \succ_{1} 4 \succ_{1} 1 \succ_{1} 2 \\
& 3 \succ_{2} 1 \sim_{2} 4 \succ_{2} 2 \succ_{2} 5 \\
& 1 \succ_{3} 5 \succ_{3} 3 \succ_{3} 2 \succ_{3} 4 \\
& 2 \succ_{4} 5 \succ_{4} 3 \succ_{4} 4 \succ_{4} 1 \\
& 2 \succ_{5} 4 \succ_{5} 5 \succ_{5} 1 \succ_{5} 3
\end{aligned}
$$

For player 3 the most preferred set is $\{1,3\}$. For player 1 , there are two most preferred sets, $\{1,3\}$ and $\{1,5\}$. However, $\{1,5\}$ cannot belong to any strict core partition, because $\{5\}$ blocks it. Hence any $\mathscr{B}$-strict core partition must contain the set $\{1,3\}$. Altogether there are three possibilities, taking into account that 5 is unacceptable for 2 :

$$
\begin{aligned}
& \mathscr{H}_{1}=\{\{1,3\},\{4,5\},\{2\}\} \\
& \mathscr{H}_{2}=\{\{1,3\},\{2,4\},\{5\}\} \\
& \mathscr{H}_{3}=\{\{1,3\},\{2,4,5\}\}
\end{aligned}
$$

However, $\mathscr{M}_{3}$ is blocked by $\{2,4\}, \mathscr{M}_{2}$ is weakly blocked by $\{4,5\}$ and $\mathscr{M}_{1}$ is weakly blocked by $\{2,4\}$. Hence there exist no $\mathscr{B}$-strict core partitions, although $\mathscr{M}_{1}$ and $\mathscr{M}_{2}$ are in the $\mathscr{B}$-core.

Algorithm BSTABLE applied to this preference profile can output anyone of $\mathscr{M}_{2}, \mathscr{H}_{3}$ and $\mathscr{M}_{4}=\{\{1,2,3,5\},\{4\}\}$, according to the order of players 
chosen in Step 2 of the algorithm and how the indifferences of players 1,2 and 4 are resolved in the course of the algorithm.

An example of a preference profile with no $\mathscr{\not B}$-core partition is given in [6].

Example 2. This example shows a case when 3 -strict core contains more than one partition, even in the case without indifferences. Let $n$ be even and let the first choice of each player $i$ be the player $i+1$ and her second choice $i-1$ (modulo $n$ ). Then in addition to the grand coalition (which will be obtained by algorithm BSTABLE) also e.g. the partition $\mathscr{M}$ consisting of pairs $\{i, i+1\}$, $i=1,3, \ldots, n-1$ is in $\mathscr{B}$-strict core. To see this, realize that for $i=1,3, \ldots$, $n-1$, player $i$ has obtained in $\mathscr{M}$ the best set in her ranking and for $i+1$, $i=1,3, \ldots, n-1$ only those sets could be better, which contain $i+2$. However, $i+2$ already has in . $M$ her best set, as remarked earlier.

A partition algorithm may be considered as a function $\phi$ assigning to each preference profile $\mathscr{P}$ a partition $\mathscr{M}$. Could a player $i$ obtain a more preferred result, if she submits to the partition algorithm a preference list $\xi_{i}^{\prime}$ different from her true preference list $\leqslant_{i}$ ? More formally. let $: \mathbb{P}^{\prime}=$ $\left(\leqslant 1, \leqslant_{2}, \ldots, \leqslant_{i}^{\prime}, \ldots, \leqslant_{n}\right)$ and let $\phi(\mathscr{P})=\mathscr{M}$ and $\phi\left(\mathscr{P}^{\prime}\right)=\mathscr{M}^{\prime}$. We say that a partition algorithm is manipulable by a player $i$ if $M^{\prime}(i) \succ_{i} M(i)$, where $\succ_{i}$ is taken with respect to the true preference list $\leqslant_{i}$. A partition algorithm is strategy-proof if it is not manipulable by any player.

Roth [10] and Bird [3] have shown that the Top-trading-cycles algorithm is strategy-proof. Their proofs carry over also to BSTẢBLE and hence we also have

Theorem 2. If all the preferences are strict, algorithm BSTABLE is strategy proof.

\section{Properties of -strict core partitions}

Theorem 3. Let all the preferences of players over individuals be strict. Let A be any partition containing a set with cardinality more than 2. Then $\mathscr{A}$ cannot be a $\mathscr{W}$-strict core partition.

Proof: Let $M \in \mathscr{M}$ be such that $|M|>2$. Take an arbitrary $i \in M$. Then the pair $\left\{i, \mathscr{B}_{i}(M)\right\}$ weakly blocks $\mathscr{M}$, since $i$ now strictly prefers this pair to the set she has been in and $\mathscr{B}_{i}(M)$ is not worse than before.

Theorem 4. Let a preference profile $\mathscr{P}$ without indifferences be given. Then all the solutions of the stable roommates problem with respect to $\mathscr{P}$ are $\mathscr{W}$-strict core partitions and hence also $\mathscr{W}$-core partitions.

Proof: We want to show that if $\mathscr{M}$ is a solution of the stable roommates problem with respect to $\mathscr{P}$, then $\mathscr{M}$ is also a $\mathscr{W}$-strict core partition. To that end suppose that $\mathscr{M}$ is not $\mathscr{W}$-strict core. Then there exists a weakly $\mathscr{W}$ blocking set $Z$. Let us consider two cases:

a) $Z=\{i\}$. Let us denote the partner of $i$ in the stable roommates solution as $j$. Then $\{i\} \succ \succ_{, i}\{i, j\}$, which means that $j$ is unacceptable for $i$, a contradiction. 
b) $|Z| \geq 2$. Let us take $i \in Z$ such that $Z \succ_{\mathscr{W},}, M(i)$. Denote $j=\mathscr{B}_{i}(Z)$ and consider $Z^{\prime}=\{i, j\} . Z^{\prime}$ is obviously also weakly $\mathscr{W}$-blocking. Let $\{i, k\}$, $\{j, l\} \in \mathscr{M}$. Since $Z^{\prime}$ is weakly $\mathscr{W}$-blocking, $Z^{\prime} \succ_{\mathscr{H}, i}\{i, k\}$ and $Z^{\prime} \geqslant \mathscr{H},\{\{j, l\}$. Definition 2 now implies $j \succ_{i} k$ and $i \geqslant_{j} l$, which can happen in one of two ways. The case $i \sim_{i} l$ means (since the preferences are strict) $i=l$, and hence $Z^{\prime}$ is one set of the partition $\mathscr{M}$, thus it cannot be weakly $\mathscr{W}^{\prime}$-blocking. If $i \succ_{j} l$, then $Z^{\prime}$ fulfills the definition of the blocking pair for the stable roommates problem, again we arrived at a contradiction.

Theorem 4 does not hold conversely, i.e. it may happen that for a given preference profile without indifferences there exists a $\mathscr{W}$-strict core partition and yet no stable roommates solution. Still a weaker form of converse holds true:

Theorem 5. Let ip be a preference profile without indifferences for the set of players $N=\{1.2 \ldots n\}$. Let Ul be any $\mathscr{W}$-strict core partition for $N$. Denote by $N^{\prime}=\{i \in N ; M(i) \geq 2\}$. Then $\mathscr{M}^{\prime}$, the restriction of $\mathscr{M}$ to $N^{\prime}$, is a stable roommates solution for : $\mathbb{P}_{N}$.

Proof: From Theorem 3 we know that the maximum size of a set in a $\mathscr{W}$-strict core partition is 2 , hence $\mathscr{M}$ partitions $N^{\prime}$ into pairs. Moreover, if a pair blocked $\mathscr{M}^{\prime}$ as a roommates solution, it would also weakly $\mathscr{W}$-block $\mathscr{M}^{\prime}$. Hence $\mathscr{M}^{\prime}$ is a stable roommates solution for $\mathscr{P}_{N^{\prime}}$.

Theorems 4 and 5 have an important consequence. Namely, in the case without indifferences, if a pair of players cannot be a stable roommates pair, it cannot be a $\mathscr{W}$-strict core pair either. Hence the stable roommates algorithm (SR algorithm for short, described in [8], Chapter 4), which successively deletes pairs that cannot be stable roommates, can be used for finding a $\mathscr{W}$-strict core partition after a small modification. On the termination of the algorithm, either a preference list of a player $i$ becomes empty, or $i$ contains a single entry $j$. In the latter case, $i$ is also the only entry in the preference list of $j$ and $\{i, j\}$ is a stable rommates pair, i.e. also a $\mathscr{W}$-strict core pair. We shall now briefly analyze what happens in the former case.

Before the actual algorithm starts, a natural step is to delete a player $i$ from the preference list of player $j$ if $j$ is not acceptable for $i$ (a preference profile obtained after all the possible reductions of this sort is called consistent). Clearly, if the prefence list of a player $i$ becomes empty after such reductions, then $\{i\}$ will not be $\mathscr{H}$-blocked, since $i$ is unacceptable for all the other players.

The Phase I of the SR algorithm deletes a pair $\left\{i^{\prime}, j\right\}$ for every $i^{\prime}$ such that $j \succ, i^{\prime}$ and $i$ is the first entry in $j$ 's preference list. If the list of $i$ becomes empty in the end this phase, again there is no player that would be willing to form a weakly blocking pair with $i$, so $\{i\}$ is a $\mathscr{W}$-strict core set

In the Phase II the so called rotations are eliminated. A sequence

$$
\rho=\left(x_{0}, y_{0}\right)\left(x_{1}, y_{1}\right) \cdots\left(x_{r-1}, y_{r-1}\right)
$$

is called a rotation if $y_{i}$ is the first choice of $x_{i}$ and $y_{i+1}$ is her second choice for $i=0,1, \ldots r-1$ (modulo $r$, when necessary). An elimination of the rotation $\rho$ means eliminating all the pairs $\left(y_{i}, z\right)$ such that $y_{i}$ prefers $x_{i-1}$ before $z$. 
If during the elimination of rotation $\rho$ the preference list of some $x_{i}$ becomes empty, then this is only possible if $x_{i}$ is also some $y_{j}$ (for a detailed argument see the proof of Lemma 4.2.7 in [8]). But then the pair $\left\{x_{i}, y_{j}\right\}$ has also been eliminated, and since $y_{j}$ was the first choice of $x_{j}$, the singleton $\left\{y_{j}\right\}$ would not be stable, since exactly the pair $\left\{x_{j}, y_{j}\right\}$ would block it.

The above observations are summarized in the following theorem:

Theorem 6. The $\mathscr{W}$-strict core of an instance of the coalition formation game without indifferences is empty if and only if the preference list of some player becomes empty during the execution of Phase II of the Stable roommates algorithm.

\section{Conclusion}

In spite of the positive result about the existence of a $\not B$-strict core partition, the question of how to describe all the 3 -ștrict core partitions remains open. Since every set of players can be blocking, the brute force approach would require an exponential number of operations. Moreover, the question whether a $\mathscr{B}$-strict core partition in the case of indiferences exists, is difficult to answer. In [5] polynomial algorithms for deciding whether a given partition belongs to $\mathscr{B}$-strict core or to $\mathscr{B}$-core have been given and in $[6]$ it has been shown that the problem of deciding about the existence of a $\mathscr{B}$-strict core and also $\mathscr{B}$-core partition in the presence of indifferences is NP-complete.

The structure of $\mathscr{W}$-core and $\mathscr{W}$-strict core partitions is studied in [7].

\section{References}

1. Alcalde J (1995) Exchange-proofness or divorce-proofness? Stability in one-sided matching markets. Economic Design 1:275-287

2. Banerjee S, Konishi H, Sönmez T (1998) Core in a simple coalition formation game. Working paper

3. Bird ChG (1984), Group incentive compatibility in a market with indivisible goods. Economics Letters 14:309-313

4. Bogomolnaia A, Jackson MO (1998) The stability of hedonic coalition structures. mimeo

5. Cechlárová K. Hajduková J (1999) Stability testing in coalition formation games. Proc. SOR'99, Eds.: Rupnik V, Zadnik Stirn L, Drobne S 111-116

6. Cechlárová K. Hajduková J (submitted) Computational complexity of Bh-core and strict . A core in coalition formation games

7. Cechlárová K, Hajduková J (in preparation) Stable partitions with $\mathscr{W}$-preferences

8. Gusfield D, Irving RW (1989) The stable marriage problem: Structure and algorithms. Foundations of Computing, MIT Press, Cambridge

9. Romero Medina A (1995) Stability in one-sided matching. Phd Thesis. Universitat Autonoma de Barcelona

10. Roth AE (1982) Incentive compatibility in a market with indivisible goods. Economics Letters 9:127 ‥132

11. Roth AE, Postlewaite A (1977) Weak versus strong domination in a market with indivisible goods. Journal of Mathematical Economics 4:131-137

12. Roth, Sotomayor M (1990) Two-sided matching: A study in game-theoretic modelling and analysis. Econometric Society Monograph Series, Cambridge University Press, New York

13. Shapley L, Scarf H (1974) On cores and indivisibility. Jour. of Math. Economics 1:23-37 